\title{
Symmetric Ground States for Doubly Nonlocal Equations with Mass Constraint
}

\author{
Silvia Cingolani ${ }^{1, * \mathbb{D}}$, Marco Gallo $^{1}(\mathbb{D})$ and Kazunaga Tanaka ${ }^{2}$ (D) \\ 1 Dipartimento di Matematica, Università degli Studi di Bari Aldo Moro—Via E. Orabona 4, 70125 Bari, Italy; \\ marco.gallo@uniba.it \\ 2 Department of Mathematics, School of Science and Engineering, Waseda University-3-4-1 Ohkubo, \\ Shijuku-ku, Tokyo 169-8555, Japan; kazunaga@waseda.jp \\ * Correspondence: silvia.cingolani@uniba.it
}

check for updates

Citation: Cingolani, S.; Gallo, M.;

Tanaka, K. Symmetric Ground States for Doubly Nonlocal Equations with Mass Constraint. Symmetry 2021, 13, 1199. https://doi.org/10.3390/ sym13071199

Academic Editor: Alexander Shapovalov

Received: 31 May 2021

Accepted: 25 June 2021

Published: 2 July 2021

Publisher's Note: MDPI stays neutral with regard to jurisdictional claims in published maps and institutional affiliations.

\begin{abstract}
We prove the existence of a spherically symmetric solution for a Schrödinger equation with a nonlocal nonlinearity of Choquard type. This term is assumed to be subcritical and satisfy almost optimal assumptions. The mass of of the solution, described by its norm in the Lebesgue space, is prescribed in advance. The approach to this constrained problem relies on a Lagrange formulation and new deformation arguments. In addition, we prove that the obtained solution is also a ground state, which means that it realizes minimal energy among all the possible solutions to the problem.
\end{abstract}

Keywords: double nonlocality; Choquard nonlinearity; Hartree term; fractional Laplacian; nonlinear Schrödinger equation; normalized solutions; symmetric solutions; Lagrange formulation; Pohozaev identity

MSC: 35B38; 35J20; 35Q40; 35Q55; 35R09; 35R11; 58E05

\section{Introduction}

In 1954, the nonlocal equation

$$
-\Delta u+\mu u=\left(\frac{1}{4 \pi|x|} *|u|^{2}\right) u \quad \text { in } \mathbb{R}^{3}
$$

was introduced by Pekar [1] in the framework of quantum mechanics, and in 1976 it arose in the work of Choquard on the modeling of an electron trapped in its own hole, in a certain approximation to the Hartree-Fock theory of one-component plasma [2] (see also [3-5]). Later, (1) was proposed by Penrose [6-9] for modeling the self-gravitational collapse of a quantum mechanical wave-function (see also [10]). The first investigations of the existence and symmetry of the solutions for Equation (1) date back to [2]. Due to its physical relevance, the existence of an infinite number of standing wave solutions to (1) with a prescribed $L^{2}$-norm was faced by Lions in [11].

Variational methods were also employed to derive the existence results of standing wave solutions for the nonlinear Choquard equation without prescribed mass [12-19]; the existence of $L^{2}$-normalized solutions was also investigated when $F(t)=|t|^{p}$ in [20] and in [21-23] for generalized Choquard nonlinearities.

In this paper, we study the existence of solutions to the nonlocal problem

$$
\left\{\begin{array}{c}
(-\Delta)^{s} u+\mu u=\left(I_{\alpha} * F(u)\right) f(u) \text { in } \mathbb{R}^{N}, \\
\int_{\mathbb{R}^{N}} u^{2} d x=c, \\
(\mu, u) \in(0,+\infty) \times H_{r}^{s}\left(\mathbb{R}^{N}\right),
\end{array}\right.
$$


where $s \in(0,1), N \geq 2, \alpha \in(0, N), F \in C^{1}(\mathbb{R}, \mathbb{R})$ with $f=F^{\prime}, c>0$, and $\mu$ is a Lagrange multiplier, part of the unknowns. Here, $I_{\alpha}: \mathbb{R}^{N} \backslash\{0\} \rightarrow \mathbb{R}$ is the Riesz potential defined by

$$
I_{\alpha}(x)=A_{N, \alpha} \frac{1}{|x|^{N-\alpha}}
$$

with $A_{N, \alpha}=\frac{\Gamma\left(\frac{N-\alpha}{2}\right)}{2^{\alpha} \pi^{N / 2} \Gamma\left(\frac{\alpha}{2}\right)}$, and the symbol

$$
(-\Delta)^{s} u(x)=C_{N, s} \int_{\mathbb{R}^{N}} \frac{u(x)-u(y)}{|x-y|^{N+2 s}} d y
$$

denotes the fractional power of the Laplace operator, where $C_{N, s}=\frac{4^{s} \Gamma\left(\frac{N+2 s}{2}\right)}{\pi^{N / 2}|\Gamma(-s)|}$ and the integral is meant in the principal value sense. Finally, we indicate by

$$
H_{r}^{s}\left(\mathbb{R}^{N}\right)=\left\{u \in H^{s}\left(\mathbb{R}^{N}\right) ; u(x)=u(|x|)\right\}
$$

the subspace composed of radially symmetric functions of the fractional Sobolev space

$$
H^{s}\left(\mathbb{R}^{N}\right)=\left\{u \in L^{2}\left(\mathbb{R}^{N}\right) ; \int_{\mathbb{R}^{N}}\left|(-\Delta)^{s / 2} u\right|^{2} d x<\infty\right\}
$$

When $s \in(0,1)$, the equation in (2) is a fractional PDE, as it involves derivatives and integrals of fractional order. The fractional Laplacian operator was introduced by Laskin [24] as an extension of the classical one $(s=1)$ in the study of NLS equations, replacing the path integral over Brownian motions with Lévy flights. This operator arises naturally in many contexts and concrete applications in various fields, such as optimization, finance, crystal dislocations, charge transport in biopolymers, flame propagation, minimal surfaces, water waves, geo-hydrology, anomalous diffusion, neural systems, phase transition and Bose-Einstein condensation (see [25-30] and references therein). We refer to [31,32] for a discussion of recent developments in the description of anomalous diffusion via fractional dynamics and to $[33,34]$ for some recent applications of fractional operators to different frameworks (analysis of the amount of bromsulphthalein in the human liver, study of thermostat systems and others). Mathematically, equations involving the fractional Laplacian, together with local nonlinearities, have been largely investigated, and some fundamental contributions can be found in [35-37]. In particular, the existence and qualitative properties of the solutions for more general classes of fractional NLS equations with local source were studied in [38-41]. The mass-constrained case was, instead, recently considered in [42,43].

In the case of Hartree type nonlinearities one of the most relevant applications arises in relativistic physics, when the nonlinearity describes the short time interactions between particles. The minimization related to the problem (2) plays a fundamental role in the mathematical description of the gravitational collapse of boson stars [27,44]. Other applications can be found in quantum chemistry [45,46] (see also [47] for some orbital stability results) and in the study of graphene [48].

In this work, we consider the problem (2), which presents some nonlocal characteristics in the source, as well as in the fractional diffusion.

In particular we assume

(f1) $f \in C(\mathbb{R}, \mathbb{R})$;

(f2) there exists $C>0$ such that for every $s \in \mathbb{R}$,

$$
|t f(t)| \leq C\left(|t|^{\frac{N+\alpha}{N}}+|t|^{\frac{N+\alpha+2 s}{N}}\right) ;
$$


(f3) $F(t)=\int_{0}^{t} f(\tau) d \tau$ satisfies

$$
\lim _{t \rightarrow 0} \frac{F(t)}{|t|^{\frac{N+\alpha}{N}}}=0, \quad \lim _{t \rightarrow+\infty} \frac{F(t)}{|t|^{\frac{N+\alpha+2 s}{N}}}=0 ;
$$

(f4) there exists $t_{0} \in \mathbb{R}, t_{0} \neq 0$ such that $F\left(t_{0}\right) \neq 0$.

We remark that the exponent $\frac{N+\alpha+2 s}{N}$ appears as an $L^{2}$-critical exponent for the fractional Choquard equations and the conditions (f1)-(f4) correspond to $L^{2}$-subcritical growths.

The unconstrained case was studied by [49] for a power nonlinearity and by [50] in the case of combined local and nonlocal power-type nonlinearities; see also [51-53].

For the general class of nonlinearities of the Berestycki-Lions type [18,54], satisfying (f1)-(f4), we introduce a Lagrangian formulation in order to obtain $L^{2}$-normalized solutions of the nonlocal problem (2) in the spirit of [42], where it is applied for fractional NLS equations with a local source (see also [55]). Namely, set $\mathbb{R}_{+}=(0,+\infty)$, a radially symmetric solution $(\mu, u) \in \mathbb{R}_{+} \times H_{r}^{s}\left(\mathbb{R}^{N}\right)$ of (2) corresponds to a critical point of the functional $\mathcal{T}^{c}: \mathbb{R}_{+} \times H_{r}^{s}\left(\mathbb{R}^{N}\right) \rightarrow \mathbb{R}$ defined by

$$
\mathcal{T}^{c}(\mu, u)=\frac{1}{2} \int_{\mathbb{R}^{N}}\left|(-\Delta)^{s / 2} u\right|^{2} d x-\frac{1}{2} \int_{\mathbb{R}^{N}}\left(I_{\alpha} * F(u)\right) F(u) d x+\frac{\mu}{2}\left(\|u\|_{2}^{2}-c\right) .
$$

Using a new variant of the Palais-Smale condition [55,56], which takes into account the Pohozaev identity, we will prove a deformation theorem which enables us to detect mini-max structures in the product space $\mathbb{R}_{+} \times H_{r}^{s}\left(\mathbb{R}^{N}\right)$, by means of a Pohozaev mountain. As stressed in [42], our deformation arguments show that solutions without Pohozaev identity are suitably deformable, and thus they do not influence the topology of the sublevels of the functional. This information could be relevant in a fractional framework since it is not known if the Pohozaev identity holds for general continuous $f$ and general values of $s \in(0,1)$.

We state our main results.

Theorem 1. Suppose $N \geq 2$, and (f1)-(f4). Then there exists $c_{0} \geq 0$ such that, for any $c>c_{0}$, the problem (2) has a radially symmetric solution.

Theorem 2. Suppose $N \geq 2$, and (f1)-(f4) together with an $L^{2}$-subcritical growth at zero, i.e.,

(f5) $\lim _{t \rightarrow 0} \frac{F(t)}{|t| \frac{N+\alpha+2 s}{N}}=+\infty$.

Then, for any $c>0$, the problem (2) has a radially symmetric solution.

We naively notice that (f5) automatically implies (f4). We remark that, as in the local unconstrained case [57], the Mountain Pass solutions obtained in the above theorems are ground state solutions, that is, they have the least energy among all solutions; see Section 6 for details. This fact gives a strong indication of the stability properties of the found solution $[47,58]$.

Remark 1. We highlight that we assume a priori the positivity of the Lagrange multiplier $\mu$ in (2). As a matter of fact, this condition seems to be quite natural: indeed, if $u$ is a ground state on the sphere $\int_{\mathbb{R}^{N}} u^{2} d x=c$, and its energy is negative, then a posteriori the corresponding Lagrange multiplier $\mu$ is strictly positive (see Proposition 5). In addition, from a physical perspective, in the study of standing waves the multiplier $\mu$ describes the frequency of the particle, and thus it is positive; moreover, this prescribed sign is characteristic also of chemical potentials in the description of ideal gases, see $[59,60]$.

The paper is organized as follows. In Section 2, we introduce the functionals and the main features on the fractional setting and the nonlocal nonlinearity, while in Section 3, we introduce the Pohozaev mountain and gain some important asymptotic results on the 
Mountain Pass level. Then, in Section 4, a weaker version of the Palais-Smale condition, modeled on the Pohozaev identity, is obtained together with a deformation theorem. Section 5 is devoted to the proofs of Theorems 1 and 2. Finally, in Section 6, we show the equivalence of our Mountain Pass approach with the minimization approach.

\section{Functional Setting}

Let $N>2 s$. In what follows, we use the notation:

$$
\begin{aligned}
& B\left(x_{0}, R\right)=\left\{x \in \mathbb{R}^{N} ;\left|x-x_{0}\right|<R\right\}, \\
& \|u\|_{r}=\left(\int_{\mathbb{R}^{N}}|u|^{r} d x\right)^{1 / r} \quad \text { for } r \in[1, \infty) .
\end{aligned}
$$

We recall the following generalized Hardy-Littlewood-Sobolev inequality [61].

Proposition 1. Let $\alpha \in(0, N)$, and let $r, h \in(1,+\infty)$ be such that $\frac{1}{r}-\frac{1}{h}=\frac{\alpha}{N}$. Then the map

$$
L^{r}\left(\mathbb{R}^{N}\right) \rightarrow L^{h}\left(\mathbb{R}^{N}\right) ; f \mapsto I_{\alpha} * f
$$

is continuous. In particular, if $r, t \in(1,+\infty)$ verify $\frac{1}{r}+\frac{1}{t}=\frac{N+\alpha}{N}$, then there exists a constant $C=C(N, \alpha, r, t)>0$ such that

$$
\left|\int_{\mathbb{R}^{N}}\left(I_{\alpha} * g\right) h d x\right| \leq C\|g\|_{r}\|h\|_{t}
$$

for all $g \in L^{r}\left(\mathbb{R}^{N}\right)$ and $h \in L^{t}\left(\mathbb{R}^{N}\right)$.

We endow the space $H^{s}\left(\mathbb{R}^{N}\right)$ and its subspace $H_{r}^{s}\left(\mathbb{R}^{N}\right)$ of radially symmetric functions with the norm

$$
\|u\|_{H_{r}^{s}}^{2}=\int_{\mathbb{R}^{N}}\left(\left|(-\Delta)^{s / 2} u\right|^{2}+u^{2}\right) d x .
$$

Recall the fractional Sobolev critical exponent

$$
2_{s}^{*}=\frac{2 N}{N-2 s}
$$

In [62], Lions proved that

$$
H_{r}^{s}\left(\mathbb{R}^{N}\right) \hookrightarrow \hookrightarrow L^{q}\left(\mathbb{R}^{N}\right)
$$

whenever $N \geq 2$ and $q \in\left(2,2_{s}^{*}\right)$. However, as shown in [63] for general $0<s \leq \frac{1}{2}$, a result in the spirit of Radial Lemma by Strauss is not available in a fractional framework.

Moreover, we recall the fractional version of the Gagliardo-Nirenberg inequality [64]

$$
\|u\|_{r} \leq C\left\|(-\Delta)^{s / 2} u\right\|_{2}^{\beta}\|u\|_{2}^{1-\beta}
$$

for $u \in H^{s}\left(\mathbb{R}^{N}\right), r \in\left[2,2_{s}^{*}\right]$ and $\beta$ satisfying

$$
\frac{1}{r}=\frac{\beta}{2_{s}^{*}}+\frac{1-\beta}{2}
$$

In what follows, we will often denote, by $q$, the lower Hardy-Littlewood-Sobolev critical exponent and, by $p$, the $L^{2}$-critical exponent appearing in (f2)-(f3), i.e.,

$$
q=\frac{N+\alpha}{N}, \quad p=\frac{N+\alpha+2 s}{N} .
$$


Remark 2. We observe that, defining the Riesz potential by $x \mapsto \frac{A_{N, \beta}}{|x|^{\beta}}$, as some authors do, we have that the critical exponents $\frac{N+\alpha}{N}<\frac{N+\alpha+2 s}{N}<\frac{N+\alpha}{N-2 s}$ become respectively $\frac{2 N-\beta}{N}<\frac{2 N-\beta+2 s}{N}<$ $\frac{2 N-\beta}{N-2 s}$.

We consider the functional $\mathcal{T}^{c}: \mathbb{R}_{+} \times H_{r}^{s}\left(\mathbb{R}^{N}\right) \rightarrow \mathbb{R}$ defined by

$$
\mathcal{T}^{c}(\mu, u)=\frac{1}{2} \int_{\mathbb{R}^{N}}\left|(-\Delta)^{s / 2} u\right|^{2} d x-\frac{1}{2} \mathcal{D}(u)+\frac{\mu}{2}\left(\|u\|_{2}^{2}-c\right),
$$

where we set

$$
\mathcal{D}(u)=\int_{\mathbb{R}^{N}}\left(I_{\alpha} * F(u)\right) F(u) d x
$$

Using Proposition 1 , it is easy to see that $\mathcal{T}^{c} \in C^{1}\left(\mathbb{R}_{+} \times H_{r}^{s}\left(\mathbb{R}^{N}\right), \mathbb{R}\right)$. We notice that $(\mu, u) \in \mathbb{R}_{+} \times H_{r}^{s}\left(\mathbb{R}^{N}\right)$ solves problem (2) if, and only if, $\partial_{u} \mathcal{T}^{c}(\mu, u)=0$ and $\partial_{\mu} \mathcal{T}^{c}(\mu, u)=0$.

Moreover we define the functional $\mathcal{J}: \mathbb{R}_{+} \times H_{r}^{s}\left(\mathbb{R}^{N}\right) \rightarrow \mathbb{R}$ by setting

$$
\mathcal{J}(\mu, u)=\frac{1}{2} \int_{\mathbb{R}^{N}}\left|(-\Delta)^{s / 2} u\right|^{2} d x-\frac{1}{2} \mathcal{D}(u)+\frac{\mu}{2}\|u\|_{2}^{2}
$$

For a fixed $\mu>0, u$ is the critical point of $\mathcal{J}(\mu, \cdot)$ means that $u$ solves

$$
\left\{\begin{array}{c}
(-\Delta)^{s} u+\mu u=\left(I_{\alpha} * F(u)\right) f(u) \text { in } \mathbb{R}^{N}, \\
u \in H_{r}^{s}\left(\mathbb{R}^{N}\right),
\end{array}\right.
$$

in the weak sense. It is immediate that

$$
\mathcal{T}^{c}(\mu, u)=\mathcal{J}(\mu, u)-\frac{\mu}{2} c
$$

Observing that

$$
H_{r}^{s}\left(\mathbb{R}^{N}\right)=\operatorname{Fix}(\mathrm{O}(N))=\left\{u \in H^{s}\left(\mathbb{R}^{N}\right) \mid \tau(Q, u)=u \text { for each } Q \in \mathrm{O}(N)\right\},
$$

where $\mathrm{O}(N)$ is the orthogonal group of rotation matrices and the isometric action is given by

$$
\tau:(Q, \mu, u) \mapsto(\mu, u(Q \cdot)) ; \mathrm{O}(N) \times\left(\mathbb{R}_{+} \times H^{S}\left(\mathbb{R}^{N}\right)\right) \rightarrow \mathbb{R}_{+} \times H^{S}\left(\mathbb{R}^{N}\right),
$$

and observed that $\mathcal{T}^{c}$, as well as $\mathcal{J}$, is $\mathrm{O}(N)$-invariant, we have by the Principle of Symmetric Criticality of Palais [65] (see also [66]) that every critical point of $\mathcal{T}^{c}$ (resp. $\mathcal{J}$ ) restricted to $\mathbb{R}_{+} \times H_{r}^{S}\left(\mathbb{R}^{N}\right)$ is actually a critical point of $\mathcal{T}^{\mathcal{c}}($ resp. $\mathcal{J})$ on the whole $\mathbb{R}_{+} \times H^{S}\left(\mathbb{R}^{N}\right)$. This observation justifies our restriction onto the radial setting.

Finally, we recall that $C^{2}$-solutions to (6) satisfy the Pohozaev identity (see ([53], Proposition 2) and ([49], Equation (6.1)))

$$
\frac{N-2 s}{2}\left\|(-\Delta)^{s / 2} u\right\|_{2}^{2}+\frac{N}{2} \mu\|u\|_{2}^{2}=\frac{N+\alpha}{2} \mathcal{D}(u)
$$

Inspired by this identity, we also introduce the Pohozaev functional $\mathcal{P}: \mathbb{R}_{+} \times$ $H_{r}^{s}\left(\mathbb{R}^{N}\right) \rightarrow \mathbb{R}$ by setting

$$
\mathcal{P}(\mu, u)=\frac{N-2 s}{2}\left\|(-\Delta)^{s / 2} u\right\|_{2}^{2}-\frac{N+\alpha}{2} \mathcal{D}(u)+\frac{N}{2} \mu\|u\|_{2}^{2}
$$


which will be used to model both the Palais-Smale condition and the geometry of the problem. We also note that

$$
\mathcal{J}(\mu, u(\cdot / t))=\frac{1}{2} t^{N-2 s}\left\|(-\Delta)^{s / 2} u\right\|_{2}^{2}-\frac{1}{2} t^{N+\alpha} \mathcal{D}(u)+\frac{1}{2} \mu t^{N}\|u\|_{2}^{2}
$$

and $\left.\frac{d}{d t}\right|_{t=1} \mathcal{J}(\mu, u(\cdot / t))=\mathcal{P}(\mu, u)$.

\section{Asymptotic Geometry}

For a fixed $\mu>0$ we introduce the set of paths

$$
\Gamma_{\mu}=\left\{\gamma \in C\left([0,1], H_{r}^{s}\left(\mathbb{R}^{N}\right)\right) ; \gamma(0)=0, \mathcal{J}(\mu, \gamma(1))<0\right\}
$$

and the Mountain Pass (MP for short) value

$$
l(\mu)=\inf _{\gamma \in \Gamma \mu} \max _{t \in[0,1]} \mathcal{J}(\mu, \gamma(t)) .
$$

Moreover we set

$$
\Sigma=\left\{(\mu, u) \in \mathbb{R}_{+} \times H_{r}^{s}\left(\mathbb{R}^{N}\right) ; \mathcal{P}(\mu, u)>0\right\} \cup\{(\mu, 0) ; \mu>0\},
$$

and observe

$$
\{(\mu, 0) ; \mu>0\} \subset \operatorname{int}(\Sigma),
$$

so that the Pohozaer mountain is given by

$$
\partial \Sigma=\left\{(\mu, u) \in \mathbb{R}_{+} \times H_{r}^{s}\left(\mathbb{R}^{N}\right) ; \mathcal{P}(\mu, u)=0, u \neq 0\right\} .
$$

Here, the boundary of $\Sigma$ is made with respect to the topology relative to the set $\mathbb{R}_{+} \times H_{r}^{s}\left(\mathbb{R}^{N}\right)$.

We prove the following proposition.

Proposition 2. Assume (f1)-(f4). Then $\Gamma_{\mu} \neq \varnothing$ and $\partial \Sigma \neq \varnothing$. Moreover

(i) $\mathcal{J}(\mu, u) \geq 0$ for all $(\mu, u) \in \Sigma$.

(ii) $\mathcal{J}(\mu, u) \geq l(\mu)>0$ for all $(\mu, u) \in \partial \Sigma$.

Proof. By exploiting (f4) and arguing as in ([18], Proposition 2.1), we obtain the existence of a function $u \in H_{r}^{s}\left(\mathbb{R}^{N}\right)$ such that $\mathcal{D}(u)>0$. Thus defined $\gamma(t)=u(\cdot / t)$ for $t>0$ and $\gamma(0)=0$ we have

- $\quad \mathcal{J}(\mu, \gamma(t))<0$ for $t$ large and $\mathcal{J}(\mu, \gamma(t))>0$ for $t$ small;

- $\quad \mathcal{P}(\mu, \gamma(t))<0$ for $t$ large and $\mathcal{P}(\mu, \gamma(t))>0$ for $t$ small.

The first claim ensures, after a suitable rescaling, that $\gamma \in \Gamma_{\mu}$, and, in particular, $l(\mu)$ is well defined and strictly positive. The second claim instead ensures, by the intermediate value theorem, that there exists a $t^{*}$, such that $\mathcal{P}\left(\mu, \gamma\left(t^{*}\right)\right)=0$, and thus $\left(\mu, \gamma\left(t^{*}\right)\right) \in \partial \Sigma$. (i) We notice that for all $(\mu, u) \in \Sigma$

$$
\mathcal{J}(\mu, u) \geq \mathcal{J}(\mu, u)-\frac{\mathcal{P}(\mu, u)}{N+\alpha}=\frac{\alpha+2 s}{2(N+\alpha)}\left\|(-\Delta)^{s / 2} u\right\|_{2}^{2}+\frac{\alpha \mu}{2(N+\alpha)}\|u\|_{2}^{2}
$$

and thus $(i)$ follows.

(ii) Let $(\mu, u) \in \partial \Sigma$, and observe that $\mathcal{D}(u)>0$. We define again $\gamma(t)=u(\cdot / t)$ so that $t \in(0,+\infty) \mapsto \mathcal{J}(\mu, \gamma(t))$ is negative for large values of $t$, and it attains the maximum in $t=1$. After a suitable rescaling, we have $\gamma \in \Gamma_{\mu}$ and thus

$$
\mathcal{J}(\mu, u)=\max _{t \in[0,1]} \mathcal{J}(\mu, \gamma(t)) \geq l(\mu)
$$


which is the claim.

To see that $\mathcal{T}^{c}(\mu, u)=\mathcal{J}(\mu, u)-\frac{\mu}{2} c$ has a MP geometry in $\mathbb{R}_{+} \times H_{r}^{s}\left(\mathbb{R}^{N}\right)$, it is crucial to analyze the behavior of $l(\mu)$ as $\mu \rightarrow+\infty$.

Lemma 1. Assume (f1)-(f4). Then,

$$
\lim _{\mu \rightarrow+\infty} \frac{l(\mu)}{\mu}=+\infty
$$

Proof. We recall $p=\frac{N+\alpha+2 s}{N}$ and $q=\frac{N+\alpha}{N}$. By (f3), for any $\delta>0$, there exists $C_{\delta}>0$ such that

$$
|F(t)| \leq \delta|t|^{p}+C_{\delta}|t|^{q} \quad \text { for all } t \in \mathbb{R} .
$$

For $v \in H_{r}^{s}\left(\mathbb{R}^{N}\right)$, setting $u_{t}=t^{N / 2} v(t \cdot)$, we have

$$
\begin{aligned}
\mathcal{D}\left(u_{t}\right) & =\mathcal{D}\left(t^{N / 2} v(t x)\right)=t^{-N-\alpha} \mathcal{D}\left(t^{N / 2} v(x)\right) \\
& \leq t^{-N-\alpha} \int_{\mathbb{R}^{N}}\left(I_{\alpha} *\left(\delta t^{\frac{N}{2} p}|v|^{p}+C_{\delta} t^{\frac{N}{2} q}|v| q\right)\right)\left(\delta t^{\frac{N}{2} p}|v|^{p}+C_{\delta} t^{\frac{N}{2} q}|v|^{q}\right) d x \\
& =t^{2 s} \int_{\mathbb{R}^{N}}\left(I_{\alpha} *\left(\delta|v|^{p}+C_{\delta} t^{-s}|v|^{q}\right)\right)\left(\delta|v|^{p}+C_{\delta} t^{-s}|v|^{q}\right) d x \\
& \equiv t^{2 s} D_{\delta, C_{\delta} t^{-s}}(v) .
\end{aligned}
$$

Here, we write for $\delta>0$ and $A \geq 0$,

$$
\begin{aligned}
& D_{\delta, A}(v)=\int_{\mathbb{R}^{N}}\left(I_{\alpha} *\left(\delta|v|^{p}+A|v|^{q}\right)\right)\left(\delta|v|^{p}+A|v|^{q}\right) d x, \\
& \mathcal{J}_{\delta, A}(v)=\frac{1}{2}\left\|(-\Delta)^{s / 2} v\right\|_{2}^{2}+\frac{1}{2}\|v\|_{2}^{2}-\frac{1}{2} D_{\delta, A}(v) .
\end{aligned}
$$

We also denote by $b(\delta, A)$ the MP value of $\mathcal{J}_{\delta, A}$. Taking the continuity and monotonicity property of $b(\delta, A)$ into account, with respect to each variable $\delta$ and $A$, and noting that $\mathcal{J}_{\delta, A}$ satisfies the Palais-Smale condition, we have

$$
\begin{gathered}
b(\delta, A) \rightarrow b(\delta, 0) \quad \text { as } A \rightarrow 0^{+}, \\
b(\delta, 0) \rightarrow+\infty \quad \text { as } \delta \rightarrow 0^{+} .
\end{gathered}
$$

Thus, we have from (12) that

$$
\mathcal{J}\left(\mu, u_{t}\right) \geq t^{2 s}\left(\frac{1}{2}\left\|(-\Delta)^{s / 2} v\right\|_{2}^{2}+\frac{1}{2} \mu t^{-2 s}\|v\|_{2}^{2}-\frac{1}{2} D_{\delta, C_{\delta} t^{-s}}(v)\right) .
$$

Setting $t=\mu^{1 / 2 s}$, we have

$$
\mathcal{J}\left(\mu, u_{\mu^{1 / 2 s}}\right) \geq \mu \mathcal{J}_{\delta, C_{\delta} \mu^{-1 / 2}}(v)
$$

and thus

$$
\frac{l(\mu)}{\mu} \geq b\left(\delta, C_{\delta} \mu^{-1 / 2}\right) .
$$

Therefore, we gain

$$
\liminf _{\mu \rightarrow+\infty} \frac{l(\mu)}{\mu} \geq \lim _{A \rightarrow 0^{+}} b(\delta, A)=b(\delta, 0) .
$$

Since $\delta>0$ is arbitrary, we obtain the claim for $\delta \rightarrow 0^{+}$. 
Corollary 1. We have

$$
B_{c} \equiv \inf _{(\mu, u) \in \partial \Sigma} \mathcal{T}^{c}(\mu, u)>-\infty
$$

Proof. By (ii) of Proposition 2 and Lemma 1, we have

$$
\inf _{(\mu, u) \in \partial \Sigma} \mathcal{T}^{c}(\mu, u) \geq \inf _{\mu>0}\left(l(\mu)-\frac{\mu}{2} c\right)>-\infty
$$

When the nonlinearity is $L^{2}$-subcritical in the origin, we are able to also prove the following behavior of $l(\mu)$ as $\mu \rightarrow 0^{+}$.

Proposition 3. Assume (f5) in addition to (f1)-(f4). Then,

$$
\lim _{\mu \rightarrow 0^{+}} \frac{l(\mu)}{\mu}=0
$$

Proof. We fix $u \in H_{r}^{s}\left(\mathbb{R}^{N}\right) \cap L^{\infty}\left(\mathbb{R}^{N}\right)$ with $\|u\|_{\infty}=1$. We note that there exists $L_{\sigma}>0$, such that

$$
\begin{gathered}
F(\sigma u(x)) \geq \sqrt{L_{\sigma}} \sigma^{p}|u(x)|^{p} \quad \text { for all } \sigma \in(0,1] \text { and } x \in \mathbb{R}^{N}, \\
L_{\sigma} \rightarrow \infty \quad \text { as } \sigma \rightarrow 0 .
\end{gathered}
$$

Recalling $D_{1,0}(u)=\int_{\mathbb{R}^{N}}\left(I_{\alpha} *|u|^{p}\right)|u|^{p} d x$, we have for $t>0$

$$
\begin{aligned}
& \mathcal{J}(\mu, \sigma u(x / t)) \leq \frac{1}{2} \sigma^{2} t^{N-2 s}\left\|(-\Delta)^{s / 2} u\right\|_{2}^{2}+\frac{\mu}{2} \sigma^{2} t^{N}\|u\|_{2}^{2}-\frac{1}{2} L_{\sigma} \sigma^{2 p} t^{N+\alpha} D_{1,0}(u) \\
& =\mu^{-\frac{N-2 s}{2 s}}\left(\frac{1}{2} \sigma^{2} \tau^{N-2 s}\|\nabla u\|_{2}^{2}+\frac{1}{2} \sigma^{2} \tau^{N}\|u\|_{2}^{2}-\frac{1}{2} L_{\sigma} \mu^{\frac{N-2 s}{2 s}} \mu^{-\frac{N+\alpha}{2 s}} \sigma^{2 p} \tau^{N+\alpha} D_{1,0}(u)\right) \\
& =\mu^{-\frac{N-2 s}{2 s}} \sigma^{2}\left(\frac{1}{2}\|\nabla u\|_{2}^{2} \tau^{N-2 s}+\frac{1}{2}\|u\|_{2}^{2} \tau^{N}-\frac{1}{2} L_{\sigma} \mu^{-\frac{2+\alpha}{2 s}} \sigma^{2 p-2} D_{1,0}(u) \tau^{N+\alpha}\right),
\end{aligned}
$$

where we set $t=\mu^{-\frac{1}{2 s}} \tau$. Moreover setting $\sigma=\mu^{\frac{N}{4 s}}$, we have

$$
\mathcal{J}\left(\mu, \mu^{\frac{N}{4 s}} u\left(x /\left(\mu^{-\frac{1}{2 s}} \tau\right)\right) \leq \mu\left(\frac{1}{2}\left\|(-\Delta)^{s / 2} u\right\|_{2}^{2} \tau^{N-2 s}+\frac{1}{2}\|u\|_{2}^{2} \tau^{N}-\frac{1}{2} L_{\mu^{N / 4 s}} D_{1,0}(u) \tau^{N+\alpha}\right) .\right.
$$

For $\mu \in(0,1)$, the map

$$
\tau \mapsto \mu^{\frac{N}{4 s}} u\left(x / \mu^{-\frac{1}{2 s}} \tau\right) ;(0,+\infty) \rightarrow H_{r}^{s}\left(\mathbb{R}^{N}\right)
$$

can be regarded as a path in $\Gamma_{\mu}$. Thus

$$
\frac{l(\mu)}{\mu} \leq \max _{\tau \in(0,+\infty)}\left(\frac{1}{2}\left\|(-\Delta)^{s / 2} u\right\|_{2}^{2} \tau^{N-2 s}+\frac{1}{2}\|u\|_{2}^{2} \tau^{N}-\frac{1}{2} L_{\mu^{N / 4 s}} D_{1,0}(u) \tau^{N+\alpha}\right) .
$$

Since $L_{\mu^{N / 4 s}} \rightarrow+\infty$ as $\mu \rightarrow 0^{+}$, we have

$$
\text { R.H.S. } \rightarrow 0 \text { as } \mu \rightarrow 0^{+} \text {. }
$$

Thus, we have the conclusion.

\section{Palais-Smale-Pohozaev Condition}

Under the assumptions (f1)-(f4), it seems hard to verify the standard Palais-Smale condition for the functional $\mathcal{T}^{c}$. As in [42], we introduce a compactness condition which is 
weaker than the standard Palais-Smale one (see also $[55,56,67,68])$. Namely, we provide the following definition, recalled as $\mathbb{R}_{+} \equiv(0,+\infty)$.

Definition 1. For $b \in \mathbb{R}$, we say that $\mathcal{T}^{c}$ satisfies the Palais-Smale-Pohozaev condition at level $b$ (in short, the $(P S P)_{b}$ condition), if for any sequence $\left(\mu_{j}, u_{j}\right) \subset \mathbb{R}_{+} \times H_{r}^{s}\left(\mathbb{R}^{N}\right)$, such that

$$
\begin{aligned}
& \mathcal{T}^{c}\left(\mu_{j}, u_{j}\right) \rightarrow b, \\
& \mu_{j} \cdot \partial_{\mu} \mathcal{T}^{c}\left(\mu_{j}, u_{j}\right) \rightarrow 0, \\
& \partial_{u} \mathcal{T}^{c}\left(\mu_{j}, u_{j}\right) \rightarrow 0 \text { strongly in }\left(H_{r}^{s}\left(\mathbb{R}^{N}\right)\right)^{*}, \\
& \mathcal{P}\left(\mu_{j}, u_{j}\right) \rightarrow 0,
\end{aligned}
$$

it happens that $\left(\mu_{j}, u_{j}\right)$ has a strongly convergent subsequence in $\mathbb{R}_{+} \times H_{r}^{s}\left(\mathbb{R}^{N}\right)$.

Remark 3. The $(P S P)_{b}$ condition introduced in Definition 1 looks slightly different from the one in [42]. We emphasize that $\mathbb{R}_{+} \times H_{r}^{s}\left(\mathbb{R}^{N}\right)$ with the standard metric induced by $\mathbb{R} \times H_{r}^{s}\left(\mathbb{R}^{N}\right)$ is not complete, and is not suitable for the deformation argument. Since $\left(\mathbb{R}_{+}, \frac{1}{x^{2}} d x^{2}\right)$ is complete, it is natural to introduce a related metric on $\mathbb{R}_{+} \times H_{r}^{s}\left(\mathbb{R}^{N}\right)$, that is, we regard $M=\mathbb{R}_{+} \times H_{r}^{s}\left(\mathbb{R}^{N}\right)$ as a Riemannian manifold with the metric

$$
\left(\left(v_{1}, w_{1}\right),\left(v_{2}, w_{2}\right)\right)_{T_{(\mu, u)} M}=\frac{1}{\mu^{2}} v_{1} v_{2}+\left(w_{1}, w_{2}\right)_{H_{r}^{s}}
$$

for $\left(v_{1}, w_{1}\right),\left(v_{2}, w_{2}\right) \in T_{(\mu, u)} M,(\mu, u) \in M$. It is easy to see that $\left(M,(\cdot, \cdot)_{T M}\right)$ is a complete Riemannian manifold. We regard $\mathcal{T}^{c}$ as a functional defined on $M$. We have

$$
\left\|\left(\partial_{\mu} \mathcal{T}^{c}(\mu, u), \partial_{u} \mathcal{T}^{c}(\mu, u)\right)\right\|_{\left(T_{(\mu, u)} M\right)^{*}}^{2}=\mu^{2}\left|\partial_{\mu} \mathcal{T}^{c}(\mu, u)\right|^{2}+\left\|\partial_{u} \mathcal{T}^{c}(\mu, u)\right\|_{\left(H_{r}^{s}\right)^{*}}^{2} .
$$

Thus (15) and (16) are equivalent to

$$
\left\|\left(\partial_{\mu} \mathcal{T}^{c}(\mu, u), \partial_{u} \mathcal{T}^{c}(\mu, u)\right)\right\|_{\left(T_{(\mu, u)} M\right)^{*}} \rightarrow 0 .
$$

Moreover, setting

$$
\widetilde{\mathcal{T}}^{c}(\lambda, u)=\mathcal{T}^{c}\left(e^{\lambda}, u\right): \mathbb{R} \times H_{r}^{s}\left(\mathbb{R}^{N}\right) \rightarrow \mathbb{R},
$$

we can observe that $\left(\lambda_{j}, u_{j}\right) \subset \mathbb{R} \times H_{r}^{s}\left(\mathbb{R}^{N}\right)$ satisfies

$$
\begin{gathered}
\partial_{\lambda} \widetilde{\mathcal{T}}^{c}\left(\lambda_{j}, u_{j}\right) \rightarrow 0, \\
\left\|\partial_{u} \widetilde{\mathcal{T}}^{c}\left(\lambda_{j}, u_{j}\right)\right\|_{\left(H_{r}^{s}\right)^{*}} \rightarrow 0
\end{gathered}
$$

if and only if $\left(\mu_{j}, u_{j}\right)=\left(e^{\lambda_{j}}, u_{j}\right)$ satisfies (18).

We remark that this compactness condition takes the scaling properties of $\mathcal{T}^{c}$ into consideration through the Pohozaev functional $\mathcal{P}$. We now show the following crucial result.

Theorem 3. Assume (f1)-(f3). Let $b<0$. Then, $\mathcal{T}^{c}$ satisfies the $(P S P)_{b}$ condition on $\mathbb{R}_{+} \times$ $H_{r}^{s}\left(\mathbb{R}^{N}\right)$.

Proof. Let $b<0$ and $\left(\mu_{j}, u_{j}\right) \subset \mathbb{R} \times H_{r}^{s}\left(\mathbb{R}^{N}\right)$ be a sequence satisfying (14)-(17). First we note that, by (15), we have

$$
\mu_{j}\left(\left\|u_{j}\right\|_{2}^{2}-c\right) \rightarrow 0
$$

Step 1: $\liminf _{j \rightarrow \infty} \mu_{j}>0$ and $\left\|u_{j}\right\|_{2}^{2} \rightarrow c$. 
By (14) and (17), we have

$$
\begin{aligned}
o(1)= & \mathcal{P}\left(\mu_{j}, u_{j}\right)=\frac{N-2 s}{2}\left\|(-\Delta)^{s / 2} u_{j}\right\|_{2}^{2}+ \\
& +(N+\alpha)\left(\mathcal{T}^{c}\left(\mu_{j}, u_{j}\right)-\frac{1}{2}\left\|(-\Delta)^{s / 2} u_{j}\right\|_{2}^{2}-\frac{\mu_{j}}{2}\left(\left\|u_{j}\right\|_{2}^{2}-c\right)\right)+\frac{N}{2} \mu_{j}\left\|u_{j}\right\|_{2}^{2} \\
= & -\frac{\alpha+2 s}{2}\left\|(-\Delta)^{s / 2} u_{j}\right\|_{2}^{2}+(N+\alpha)(b+o(1))+\frac{N}{2} \mu_{j} c+o(1),
\end{aligned}
$$

Here, we have used (19). Since $b<0$, we have $\liminf _{j \rightarrow \infty} \mu_{j}>0$. Thus (19) implies $\left\|u_{j}\right\|_{2}^{2} \rightarrow c$.

Step 2: $\left\|(-\Delta)^{s / 2} u_{j}\right\|_{2}^{2}$ and $\mu_{j}$ are bounded.

Since $\varepsilon_{j} \equiv\left\|\partial_{u} \mathcal{T}^{c}\left(\mu_{j}, u_{j}\right)\right\|_{\left(H_{r}^{s}\left(\mathbb{R}^{N}\right)\right)^{*}} \rightarrow 0$, we have

$$
\left\|(-\Delta)^{s / 2} u_{j}\right\|_{2}^{2}-\int_{\mathbb{R}^{N}}\left(I_{\alpha} * F\left(u_{j}\right)\right) f\left(u_{j}\right) u_{j} d x+\mu_{j}\left\|u_{j}\right\|_{2}^{2} \leq \varepsilon_{j}\left\|u_{j}\right\|_{H_{r}^{s}} .
$$

Note that $\frac{2 N p}{N+\alpha} \in\left(2,2_{s}^{*}\right)$. Moreover, we observe that, by (f3), for $\delta>0$, when fixed, there exists $C_{\delta}>0$ such that

$$
|F(t)| \leq \delta|t|^{p}+C_{\delta}|t|^{\frac{N+\alpha}{N}}, \quad t \in \mathbb{R}
$$

where $p=\frac{N+\alpha+2 s}{N}$, and thus

$$
\left\|F\left(u_{j}\right)\right\|_{\frac{2 N}{N+\alpha}} \leq \delta\left\|\left|u_{j}\right|^{p}\right\|_{\frac{2 N}{N+\alpha}}+C_{\delta}\left\|\left|u_{j}\right|^{\frac{N+\alpha}{N}}\right\|_{\frac{2 N}{N+\alpha}}=\delta\left\|u_{j}\right\|_{\frac{2 N p}{N+\alpha}}^{p}+C_{\delta}\left\|u_{j}\right\|_{2}^{\frac{N+\alpha}{N}} .
$$

Therefore, by (f2) we have

$$
\begin{aligned}
& \int_{\mathbb{R}^{N}}\left(I_{\alpha} *\left|F\left(u_{j}\right)\right|\right)\left|f\left(u_{j}\right) u_{j}\right| d x \\
& \quad \leq C\left\|F\left(u_{j}\right)\right\|_{\frac{2 N}{N+\alpha}}\left\|f\left(u_{j}\right) u_{j}\right\|_{\frac{2 N}{N+\alpha}} \\
& \quad \leq C\left(\delta\left\|u_{j}\right\|_{\frac{2 N p}{N+\alpha}}^{p}+C_{\delta}\left\|u_{j}\right\|_{2}^{\frac{N+\alpha}{N}}\right) \cdot C^{\prime}\left(\left\|u_{j}\right\|_{\frac{2 N p}{N+\alpha}}^{p}+\left\|u_{j}\right\|_{2}^{\frac{N+\alpha}{N}}\right) \\
& \quad=C C^{\prime} \delta\left\|u_{j}\right\|_{\frac{2 N p}{N+\alpha}}^{2 p}+C C^{\prime}\left(\delta+C_{\delta}\right)\left\|u_{j}\right\|_{\frac{2 N p}{N+\alpha}}^{p}\left\|u_{j}\right\|_{2}^{\frac{N+\alpha}{N}}+C C^{\prime} C_{\delta}\left\|u_{j}\right\|_{2}^{\frac{2(N+\alpha)}{N}} \\
& =C C^{\prime} \delta\left\|u_{j}\right\|_{\frac{2 N p}{N+\alpha}}^{2 p}+C C^{\prime}\left(\delta+C_{\delta}\right)\left(\frac{\delta}{2}\left\|u_{j}\right\|_{\frac{2 N p}{N+\alpha}}^{2 p}+\frac{1}{2 \delta}\left\|u_{j}\right\|_{2}^{\frac{2(N+\alpha)}{N}}\right)+C C^{\prime} C_{\delta}\left\|u_{j}\right\|_{2}^{\frac{2(N+\alpha)}{N}} \\
& \leq C^{\prime \prime} \delta\left\|u_{j}\right\|_{\frac{2 N p}{N+\alpha}}^{2 p}+C_{\delta}^{\prime \prime}\left\|u_{j}\right\|_{2}^{\frac{2(N+\alpha)}{N}}
\end{aligned}
$$

and thus, by Gagliardo-Nirenberg inequality (4), with $r=\frac{2 N p}{N+\alpha}$ and $\beta=\frac{1}{p}$, we derive

$$
\begin{gathered}
\left\|(-\Delta)^{s / 2} u_{j}\right\|_{2}^{2}+\mu_{j}\left\|u_{j}\right\|_{2}^{2} \leq \int_{\mathbb{R}^{N}}\left(I_{\alpha} *\left|F\left(u_{j}\right)\right|\right)\left|f\left(u_{j}\right) u_{j}\right| d x+\varepsilon_{j}\left\|u_{j}\right\|_{H_{r}^{s}} \\
\leq C^{\prime \prime} \delta\left\|(-\Delta)^{s / 2} u_{j}\right\|_{2}^{2}\left\|u_{j}\right\|_{2}^{2(p-1)}+C_{\delta}^{\prime \prime}\left\|u_{j}\right\|_{2}^{\frac{2(N+\alpha)}{N}}+\varepsilon_{j}\left\|u_{j}\right\|_{H_{r}^{s}} .
\end{gathered}
$$

Since $\left\|u_{j}\right\|_{2}^{2}=c+o(1)$, we have

$$
\begin{aligned}
& \left(1-C^{\prime \prime} \delta(c+o(1))^{p-1}\right)\left\|(-\Delta)^{s / 2} u_{j}\right\|_{2}^{2}+\mu_{j}(c+o(1)) \\
& \leq C_{\delta}^{\prime \prime}(c+o(1))^{\frac{N+\alpha}{N}}+\varepsilon_{j}\left(\left\|(-\Delta)^{s / 2} u_{j}\right\|_{2}^{2}+c+o(1)\right)^{1 / 2}
\end{aligned}
$$

For a small enough $\delta$, we have a boundedness of $\left\|(-\Delta)^{s / 2} u_{j}\right\|_{2}$ and $\mu_{j}$. 
Step 3: Convergence in $\mathbb{R}_{+} \times H_{r}^{s}\left(\mathbb{R}^{N}\right)$.

By Steps $1-2$, the sequence $\left(\mu_{j}, u_{j}\right)$ is bounded in $\mathbb{R}_{+} \times H_{r}^{s}\left(\mathbb{R}^{N}\right)$ and thus, after extracting a subsequence denoted in the same way, we may assume that $\mu_{j} \rightarrow \mu_{0}>0$ and $u_{j} \rightarrow u_{0}$ weakly in $H_{r}^{s}\left(\mathbb{R}^{N}\right)$ for some $\left(\mu_{0}, u_{0}\right) \in \mathbb{R}_{+} \times H_{r}^{s}\left(\mathbb{R}^{N}\right)$.

Step 4: Conclusion.

Taking into account the assumptions (f1)-(f4), we obtain

$$
\int_{\mathbb{R}^{N}}\left(I_{\alpha} * F\left(u_{j}\right)\right) f\left(u_{j}\right) u_{0} d x \rightarrow \int_{\mathbb{R}^{N}}\left(I_{\alpha} * F\left(u_{0}\right)\right) f\left(u_{0}\right) u_{0} d x
$$

and

$$
\int_{\mathbb{R}^{N}}\left(I_{\alpha} * F\left(u_{j}\right)\right) f\left(u_{j}\right) u_{j} d x \rightarrow \int_{\mathbb{R}^{N}}\left(I_{\alpha} * F\left(u_{0}\right)\right) f\left(u_{0}\right) u_{0} d x .
$$

Thus, we derive that $\left\langle\partial_{u} \mathcal{T}^{c}\left(\mu_{j}, u_{j}\right), u_{j}\right\rangle \rightarrow 0$ and $\left\langle\partial_{u} \mathcal{T}^{c}\left(\mu_{j}, u_{j}\right), u_{0}\right\rangle \rightarrow 0$, and hence

$$
\left\|(-\Delta)^{s / 2} u_{j}\right\|_{2}^{2}+\mu_{0}\left\|u_{j}\right\|_{2}^{2} \rightarrow\left\|(-\Delta)^{s / 2} u_{0}\right\|_{2}^{2}+\mu_{0}\left\|u_{0}\right\|_{2}^{2}
$$

which implies a $u_{j} \rightarrow u_{0}$ strongly in $H_{r}^{s}\left(\mathbb{R}^{N}\right)$.

Remark 4. We emphasize that the $(P S P)_{b}$ condition does not hold at level $b=0$; it is sufficient to consider an infinitesimal sequence $\left(\mu_{j}, 0\right)$ with $\mu_{j} \rightarrow 0$.

Now, we introduce the set of critical points of $\mathcal{T}^{c}$ satisfying the Pohozaev identity, that is

$K_{b}=\left\{(\mu, u) \in \mathbb{R}_{+} \times H_{r}^{s}\left(\mathbb{R}^{N}\right) \mid \mathcal{T}^{\mathcal{c}}(\mu, u)=b, \partial_{\mu} \mathcal{T}^{\mathcal{c}}(\mu, u)=0, \partial_{u} \mathcal{T}^{\mathcal{c}}(\mu, u)=0, \mathcal{P}(\mu, u)=0\right\}$

Notice that in the definition of $K_{b}$ the condition $\mathcal{P}(\mu, u)=0$ is not trivial, since it is not known if the equality is satisfied by every solution, for general continuous $f$ and $s \in(0,1)$ (see $[49,53]$ and Proposition 5 for some particular cases). In fact, we can not generally recognize that the standard set of critical points at level $b$ is compact, and thus we restrict this to the set $K_{b}$ of critical points satisfying the Pohozaev identity.

Moreover, for each $b \in \mathbb{R}$, we introduce the following notation for sublevels

$$
\left[\mathcal{T}^{c} \leq b\right]=\left\{(\mu, u) \in \mathbb{R}_{+} \times H_{r}^{s}\left(\mathbb{R}^{N}\right) \mid \mathcal{T}^{c}(\mu, u) \leq b\right\} .
$$

Following arguments in ([56], Proposition 4.5) (see also [55], Proposition 3.1 and Corollary 4.3), we can establish the following deformation theorem for the functional $\mathcal{T}^{c}$.

Theorem 4. Assume (f1)-(f3) and $b<0$. Then, $K_{b}$ is compact in $\mathbb{R}_{+} \times H_{r}^{s}\left(\mathbb{R}^{N}\right)$ and $K_{b} \cap\left(\mathbb{R}_{+} \times\right.$ $\{0\})=\varnothing$. Moreover, for any open neighborhood $U$ of $K_{b}$ and $\bar{\varepsilon}>0$, there exists an $\varepsilon \in(0, \bar{\varepsilon})$ and a continuous map

$$
\eta(t, \mu, u):[0,1] \times \mathbb{R}_{+} \times H_{r}^{s}\left(\mathbb{R}^{N}\right) \rightarrow \mathbb{R}_{+} \times H_{r}^{s}\left(\mathbb{R}^{N}\right)
$$

such that

$\left(1^{o}\right) \eta(0, \mu, u)=(\mu, u) \quad \forall(\mu, u) \in \mathbb{R}_{+} \times H_{r}^{s}\left(\mathbb{R}^{N}\right) ;$

$\left(2^{o}\right) \eta(t, \mu, u)=(\mu, u) \quad \forall(t, \mu, u) \in[0,1] \times\left[\mathcal{T}^{c} \leq b-\bar{\varepsilon}\right] ;$

$\left(3^{o}\right) \mathcal{T}^{c}(\eta(t, \mu, u)) \leq \mathcal{T}^{c}(\mu, u) \quad \forall(t, \mu, u) \in[0,1] \times \mathbb{R}_{+} \times H_{r}^{s}\left(\mathbb{R}^{N}\right)$;

$\left(4^{o}\right) \eta\left(1,\left[\mathcal{T}^{c} \leq b+\varepsilon\right] \backslash U\right) \subset\left[\mathcal{T}^{c} \leq b-\varepsilon\right] ;$

$\left(5^{o}\right) \eta\left(1,\left[\mathcal{T}^{c} \leq b+\varepsilon\right]\right) \subset\left[\mathcal{T}^{c} \leq b-\varepsilon\right] \cup U$;

$\left(6^{o}\right)$ if $K_{b}=\varnothing$, we have $\eta\left(1,\left[\mathcal{T}^{c} \leq b+\varepsilon\right]\right) \subset\left[\mathcal{T}^{c} \leq b-\varepsilon\right]$. 


\section{Minimax Theorem}

For any $c>0$, let $B_{c}$ be the constant defined in Corollary 1. As a minimax class for $\mathcal{T}^{c}$, we define

$$
\begin{aligned}
\Lambda_{c}= & \left\{\xi \in C\left([0,1], \mathbb{R}_{+} \times H_{r}^{s}\left(\mathbb{R}^{N}\right)\right) ; \xi(0) \in \mathbb{R}_{+} \times\{0\},\right. \\
& \left.\mathcal{T}^{c}(\xi(0)) \leq B_{c}-1, \xi(1) \notin \Sigma \text { and } \mathcal{T}^{c}(\xi(1)) \leq B_{c}-1\right\} .
\end{aligned}
$$

In the following Proposition 4 we prove that $\Lambda_{c} \neq \varnothing$; moreover, by (10) we have $\xi([0,1]) \cap \partial \Sigma \neq \varnothing$ for each $\xi \in \Lambda_{c}$. Therefore from Corollary 1 , the mini-max value

$$
\beta_{c}=\inf _{\xi \in \Lambda_{c}} \max _{t \in[0,1]} \mathcal{T}^{c}(\xi(t))
$$

is well-defined and finite. Since the Palais-Smale-Pohozaev condition holds for $b \in$ $(-\infty, 0)$, it is important to estimate $\beta_{c}$. We have the following proposition.

Proposition 4. Assume (f1)-(f4). For each $c>0$ we have $\Lambda_{c} \neq \varnothing$ and

$$
\beta_{c} \leq l(\mu)-\frac{\mu}{2} c, \quad \text { for any } \mu>0 .
$$

As a consequence

(i) for a sufficiently large $c$, that is

$$
c>c_{0}=2 \inf _{\mu>0} \frac{l(\mu)}{\mu}
$$

we have $\beta_{c}<0$;

(ii) if (f5) holds, then $\beta_{c}<0$ for all $c>0$;

(iii) $\lim _{c \rightarrow+\infty} \frac{\beta_{c}}{c}=-\infty$.

Proof. (i) Let $\mu>0$ and $\gamma \in \Gamma_{\mu} \neq \varnothing$. By definition we have $\mathcal{D}(\gamma(1))>0$, thus $\mathcal{J}(\mu, \gamma(1)(\cdot / t)) \rightarrow-\infty$ and $\mathcal{P}(\mu, \gamma(1)(\cdot / t)) \rightarrow-\infty$ as $t \rightarrow+\infty$. Thus for $L \gg 0$

$$
\mathcal{T}^{c}(\mu, \gamma(1)(\cdot / L)) \leq B_{c}-1, \quad \gamma(1)(\cdot / L) \notin \Omega .
$$

We also note that $\mathcal{T}^{c}(t, 0) \rightarrow-\infty$ as $t \rightarrow+\infty$. Therefore, joining the path $\gamma_{\mu}$ with the map $t \mapsto(\mu+L t, 0)$ with a large enough $L \gg 0$, we can find a path $\xi \in \Lambda_{c}$ such that

$$
\max _{t \in[0,1]} \mathcal{T}^{c}(\xi(t)) \leq \max _{t \in[0,1]} \mathcal{J}(\mu, \gamma(t))-\frac{\mu}{2} c,
$$

and thus (22). For, $c \gg 0$ we have the claim:

(ii) If (f5) holds, we can apply Proposition 3 to obtain

$$
\lim _{\mu \rightarrow 0} \frac{l(\mu)-\frac{c}{2} \mu}{\mu}=-\frac{c}{2}<0,
$$

and, thus, the claim by (22).

(iii) Finally for any $\mu>0$ we have, by (22),

$$
\limsup _{c \rightarrow+\infty} \frac{\beta_{c}}{c} \leq \lim _{c \rightarrow+\infty}\left(\frac{l(\mu)}{c}-\frac{\mu}{2}\right)=-\frac{\mu}{2} .
$$

Since $\mu$ is arbitrary, we have (iii).

Proof of Theorems 1 and 2. Using Theorem 4, we derive that the level $\beta_{\mathcal{c}}<0$, defined in (21), is critical, and thus Theorems 1 and 2 hold. 


\section{6. $L^{2}$-Ground States}

We introduce the functional $\mathcal{L}: S_{\mathcal{c}} \rightarrow \mathbb{R}$ defined by

$$
\mathcal{L}(u)=\frac{1}{2} \int_{\mathbb{R}^{N}}\left|(-\Delta)^{s / 2} u\right|^{2} d x-\frac{1}{2} \int_{\mathbb{R}^{N}}\left(I_{\alpha} * F(u)\right) F(u) d x
$$

on the sphere

$$
S_{c}=\left\{u \in H_{r}^{s}\left(\mathbb{R}^{N}\right) ;\|u\|_{2}^{2}=c\right\} .
$$

We consider the ground state level

$$
\kappa_{c}=\inf _{u \in S_{c}} \mathcal{L}(u) .
$$

We pass this to recognize that the Mountain Pass solution found in Theorems 1 and 2 is a ground state solution, namely, a minimizer of $\mathcal{L}$ on the sphere.

Proposition 5. Assume (f1)-(f4), and let $c \geq c_{0}$, where $c_{0}$ is introduced in Proposition 4. If (f5) holds, then take $c_{0}=0$. We have that the following statements hold.

(i) Every Mountain Pass solution at level $\beta_{c}$ is a Pohozaev minimum on the product space, that is

$$
\beta_{c}=B_{c}
$$

where $B_{c}$ is defined in Corollary 1.

(ii) The Mountain Pass level and the ground state level coincide, i.e.,

$$
\kappa_{c}=\beta_{c} .
$$

In particular, thanks to Theorem 1, there exists a ground state of $\mathcal{L}_{\mid S_{c}}$.

(iii) Every ground state of $\mathcal{L}_{\mid S_{c}}$ is a solution of problem (2), i.e., the associated Lagrange multiplier is positive.

(iv) Every ground state of $\mathcal{L}_{\mid S_{c}}$ satisfies the Pohozaev identity (7) with $\mu$ the associated (positive) Lagrange multiplier. Thanks to (25), the same conclusion holds for every Mountain Pass solution at level $\beta_{c}$.

Proof. (i) From (22) and (ii) of Proposition 2 we have

$$
\beta_{c} \leq \inf _{\mu>0}\left(l(\mu)-\frac{\mu}{2} c\right) \leq B_{c} .
$$

On the other hand, each path of $\Lambda_{c}$ passes through $\partial \Sigma$ by definition, and thus

$$
\beta_{c} \geq B_{c}
$$

which gives the claim $(i)$.

(ii) Let $u_{*}$ be the Mountain Pass solution obtained in Theorems 1 and 2, which verifies $\left\|u_{*}\right\|_{2}^{2}=c$. Thus,

$$
\kappa_{c} \leq \mathcal{L}\left(u_{*}\right)=\beta_{c}<0 .
$$

In particular, by (26) we can find a minimizing sequence $\left(u_{n}\right)_{n} \subset S_{c}$ for $\kappa_{c}$ satisfying $\mathcal{L}\left(u_{n}\right)<0$, and thus we can set

$$
\mu_{n}=\frac{2}{N c}\left(\frac{\alpha+2 s}{2}\left\|(-\Delta)^{s / 2} u_{n}\right\|_{2}^{2}-(N+\alpha) \mathcal{L}\left(u_{n}\right)\right)>0
$$

so that $\mathcal{P}\left(\mu_{n}, u_{n}\right)=0$, i.e., $\left(\mu_{n}, u_{n}\right) \in \partial \Sigma$. At this point Corollary 1 and $(i)$ imply

$$
\kappa_{c}+o(1)=\mathcal{L}\left(u_{n}\right)=\mathcal{T}^{c}\left(\mu_{n}, u_{n}\right) \geq B_{c}=\beta_{c} .
$$


Passing to the limit, together with (26), we have (25).

(iii)-(iv) Let $u_{0}$ be a minimizer of $\mathcal{L}$ on $S_{c}$. Corresponding to $u_{0}$, there exists a Lagrange multiplier $\mu_{0} \in \mathbb{R}$ such that

$$
(-\Delta)^{s / 2} u_{0}+\mu_{0} u_{0}=\left(I_{\alpha} * F\left(u_{0}\right)\right) f\left(u_{0}\right),
$$

and thus, in particular,

$$
\left\|(-\Delta)^{s / 2} u_{0}\right\|_{2}^{2}+\mu_{0}\left\|u_{0}\right\|_{2}^{2}=\int_{\mathbb{R}^{N}}\left(I_{\alpha} * F\left(u_{0}\right)\right) f\left(u_{0}\right) u_{0} d x .
$$

We show first that $u_{0}$ satisfies the Pohozaev identity. In fact, we consider the $\mathbb{R}$-action $\Phi: \mathbb{R} \times S_{c} \rightarrow S_{c}$ defined by

$$
\left(\Phi_{\theta} v\right)(x)=e^{\frac{N}{2}} \theta v\left(e^{\theta} x\right),
$$

since $\left\|\Phi_{\theta} v\right\|_{2}^{2}=\|v\|_{2}^{2}$. Then we have

$$
\mathcal{L}\left(\Phi_{\theta} u_{0}\right)=\frac{1}{2} e^{2 s \theta}\left\|(-\Delta)^{s / 2} u_{0}\right\|_{2}^{2}-\frac{1}{2} e^{-(N+\alpha) \theta} \int_{\mathbb{R}^{N}}\left(I_{\alpha} * F\left(e^{\frac{N}{2} \theta} u_{0}\right)\right) F\left(e^{\frac{N}{2} \theta} u_{0}\right) d x
$$

Since $u_{0}$ is a minimizer, we have $\left.\frac{d}{d \theta}\right|_{\theta=0} \mathcal{L}\left(\Phi_{\theta} u_{0}\right)=0$, that is,

$$
s\left\|(-\Delta)^{s / 2} u_{0}\right\|_{2}^{2}+\frac{N+\alpha}{2} \int_{\mathbb{R}^{N}}\left(I_{\alpha} * F\left(u_{0}\right)\right) F\left(u_{0}\right) d x-\frac{N}{2} \int_{\mathbb{R}^{N}}\left(I_{\alpha} * F\left(u_{0}\right)\right) f\left(u_{0}\right) u_{0} d x=0 .
$$

From (27) and (29), the Pohozaev identity follows

$$
\frac{N-2 s}{2}\left\|(-\Delta)^{s / 2} u_{0}\right\|_{2}^{2}+\frac{N}{2} \mu_{0}\left\|u_{0}\right\|_{2}^{2}=\frac{N+\alpha}{2} \mathcal{D}\left(u_{0}\right) .
$$

Finally, from (26) we have $\mathcal{L}\left(u_{0}\right)=\kappa_{\mathcal{c}}<0$, that is

$$
\frac{1}{2}\left\|(-\Delta)^{s / 2} u_{0}\right\|_{2}^{2}-\frac{1}{2} \mathcal{D}\left(u_{0}\right)=\kappa_{c}<0,
$$

which joined to (30) gives $\mu_{0}>0$. This concludes the proof.

Remark 5. We notice that, by using only

$$
0>\beta_{c} \geq \kappa_{c} \geq B_{c}>-\infty
$$

and the $(P S P)_{\kappa_{c}}$ condition, we can directly obtain the existence of a minimizer $\tilde{u}$ of $\mathcal{L}$ on $S_{c}$. We provide only an outline of the proof, and refer to [42] for details. Indeed, consider again the action $\Phi_{\theta}$ on $S_{c}$ defined in (28). Set

$$
\widehat{\mathcal{L}}(\theta, u)=\mathcal{L}\left(\Phi_{\theta} u\right): \mathbb{R} \times S_{c} \rightarrow \mathbb{R},
$$

we observe

$$
\kappa_{c}=\inf _{(\theta, u) \in \mathbb{R} \times S_{c}} \widehat{\mathcal{L}}(\theta, u) .
$$

Since $\kappa_{c} \in \mathbb{R}$, applying Ekeland Principle to $\widehat{\mathcal{L}}: \mathbb{R} \times S_{c} \rightarrow \mathbb{R}$, we find a sequence $\left(\theta_{j}, u_{j}\right) \subset$ $\mathbb{R} \times S_{c}$ such that

$$
\widehat{\mathcal{L}}\left(\theta_{j}, u_{j}\right) \rightarrow \kappa_{c}, \partial_{\theta} \widehat{\mathcal{L}}\left(\theta_{j}, u_{j}\right) \rightarrow 0, \partial_{u} \widehat{\mathcal{L}}\left(\theta_{j}, u_{j}\right) \rightarrow 0 .
$$
for $\mathcal{T}^{c}$.

Setting $\hat{u}_{j}=\Phi_{\theta_{j}} u_{j}$, we observe that, for a suitable $\mu_{j}>0,\left(\mu_{j}, \hat{u}_{j}\right)$ is a $(P S P)_{\kappa_{c}}$-sequence

Thus, provided that $\kappa_{c}<0$, thanks to Theorem $3, \hat{u}_{j}$ converges up to a subsequence, to a minimizer of $\mathcal{L}$ on $S_{c}$, and, therefore, the claim. 


\section{Conclusions}

In this paper, we prove the existence of a radially symmetric solution to the nonlocal problem

$$
(-\Delta)^{s} u+\mu u=\left(I_{\alpha} * F(u)\right) f(u) \quad \text { in } \mathbb{R}^{N}
$$

coupled with the mass constraint $\int_{\mathbb{R}^{N}} u^{2} d x=c$. The result is obtained for general values of $s \in(0,1)$ and $\alpha \in(0, N)$, and by assuming quite general assumptions on the function $f$, which are almost optimal and include some particular cases such as pure powers $f(t) \sim t^{r}$, cooperating powers $f(t) \sim t^{r}+t^{h}$, competing powers $f(t) \sim t^{r}-t^{h}$ and saturable functions $f(t) \sim \frac{t^{3}}{1+t^{2}}$ (which arise, for instance, in nonlinear optics [69]).

The existence is obtained through a Lagrange formulation of the constrained problem, performing a minimax argument on a product space by means of a Pohozaev mountain. The use of the Pohozaev identity in the definition of the Palais-Smale condition allows to overcome the problem of the lack of a Pohozaev identity for general solutions, typical of the fractional framework.

Finally, we show that the Mountain Pass solution assumes the minimal energy among all the possible solutions with the same mass, which points out the physical relevance of the found solution.

$$
\begin{aligned}
& \text { When } s=\frac{1}{2}, N=3, \alpha=2 \text { and } f(t)=|t|^{r-2} t \text {, we obtain } \\
& \qquad \sqrt{-\Delta} u+\mu u=\left(\frac{1}{|x|} *|u|^{r}\right)|u|^{r-2} u \text { in } \mathbb{R}^{3} .
\end{aligned}
$$

In the $L^{2}$-critical case $r=2$, the equation is the well-known massless boson stars equation [70-72]; in this case, the quantity $\int_{\mathbb{R}^{3}} u^{2} d x=c$ represents the total $L^{2}$-mass of the body and plays a fundamental role in the study of the gravitational collapse of boson stars, where a critical value is given by the Chandrasekhar limiting mass. In this paper, we address the subcritical case $r \in\left(\frac{5}{3}, 2\right)$, but we believe that this result, together with the developed minimax tools, can be a first step towards the study of the $L^{2}$-mass critical case, since, in this case, the minimization approch is not well posed. Moreover, the high generality assumed on the function $f$ could be useful in the study of different physical problems.

Author Contributions: S.C., M.G. and K.T. have equally contributed to its elaboration. All authors have read and agreed to the published version of the manuscript.

Funding: The first and second authors are supported by PRIN 2017JPCAPN "Qualitative and quantitative aspects of nonlinear PDEs" and by INdAM-GNAMPA. The second author is supported in part by Grant-in-Aid for Scientific Research (19H00644, 18KK0073, 17H02855, 16K13771) of Japan Society for the Promotion of Science.

Conflicts of Interest: The authors declare no conflict of interest.

\section{References}

1. Pekar, S. Untersuchung über die Elektronentheorie der Kristalle; Akademie: Berlin, Germany, 1954.

2. Lieb, E.H. Existence and uniqueness of the minimizing solution of Choquard's nonlinear equation. Stud. Appl. Math. 1977, 57, 93-105. [CrossRef]

3. Fröhlich, J.; Lenzmann, E. Mean-field limit of quantum Bose gases and nonlinear Hartree equation. In Sémin. Équ. Dériv. Partielles; École Polytech.: Palaiseau, France, 2004; Talk No. 18, p. 26.

4. Fröhlich, J.; Tsai, T.-P.; Yau, H.-T. On the point-particle (Newtonian) limit of the non-linear Hartree equation. Comm. Math. Phys. 2002, 225, 223-274. [CrossRef]

5. Hartree, D. The wave mechanics of an atom with a non-Coulomb central field. Part I. Theory and methods. Math. Proc. Camb. Philos. Soc. 1928, 24, 89-110. [CrossRef]

6. Moroz, I.M.; Penrose, R.; Tod, P. Spherically-symmetric solutions of the Schrödinger-Newton equations. In Proceedings of the Topology of the Universe Conference, Cleveland, OH, USA, 17-19 October 1997; IOP Publishing: Bristol, UK; 1998; Volume 15, pp. 2733-2742.

7. Penrose, R. On gravity's role in quantum state reduction. Gen. Rel. Grav. 1996, 28, 581-600. [CrossRef] 
8. Penrose, R. Quantum computation, entanglement and state reduction. R. Soc. Lond. Philos. Trans. Ser. A Math. Phys. Eng. Sci. 1998, 356, 1927-1939. [CrossRef]

9. Penrose, R. The Road to Reality. A Complete Guide to the Laws of the Universe; Alfred A. Knopf Inc.: New York, NY, USA, 2005.

10. Tod, P. The ground state energy of the Schrödinger-Newton equation. Phys. Lett. A 2001, 280, 173-176. [CrossRef]

11. Lions, P.-L. The Choquard equation and related questions. Nonlinear Anal. 1980, 4, 1063-1073. [CrossRef]

12. Cingolani, S.; Clapp, M.; Secchi, S. Multiple solutions to a magnetic nonlinear Choquard equation. Z. Angew. Math. Phys. 2012, 63, 233-248. [CrossRef]

13. Cingolani, S.; Clapp, M.; Secchi, S. Intertwining semiclassical solutions to a Schrödinger-Newton system. Discrete Contin. Dyn. Syst. Ser. S 2013, 6, 891-908.

14. Clapp, M.; Salazar, D. Positive and sign changing solutions to a nonlinear Choquard equation. J. Math. Anal. Appl. 2013, 407, 1-15. [CrossRef]

15. Lenzmann, E. Uniqueness of ground states for pseudorelativistic Hartree equations. Anal. PDE 2009, 2, 1-27. [CrossRef]

16. Ma, L.; Zhao, L. Classification of positive solitary solutions of the nonlinear Choquard equation. Arch. Rational Mech. Anal. 2010, 195, 455-467. [CrossRef]

17. Moroz, V.; Schaftingen, J.V. Groundstates of nonlinear Choquard equations: Existence, qualitative properties and decay asymptotics. J. Funct. Anal. 2013, 265, 153-184. [CrossRef]

18. Moroz, V.; Schaftingen, J.V. Existence of groundstates for a class of nonlinear Choquard equations. Trans. Am. Math. Soc. 2015, 367, 6557-6579. [CrossRef]

19. Moroz, V.; Schaftingen, J.V. A guide to the Choquard equation. J. Fixed Point Theory Appl. 2017, 19, $773-813$.

20. Ye, H. Existence and stability waves for nonlinear fractional Schrödinger equation with Hartree type nonlinearity. J. Math. Anal. Appl. 2014, 4112, 530-542.

21. Bartsch, T.; Liu, Y.; Liu, Z. Normalized solutions for a class of nonlinear Choquard equations. SN Partial. Differ. Equ. Appl. 2020, 1, 34. [CrossRef]

22. Cingolani, S.; Tanaka, K. Ground State Solutions for the Nonlinear Choquard Equation with Prescribed Mass; to Appear on “Geometric Properties for Parabolic and Elliptic PDE's"; INdAM Springer Series; Springer: Cortona, Italy, 2019.

23. Cingolani, S.; Jeanjean, L. Stationary solutions with prescribed $L^{2}$-norm for the planar Schrödinger-Poisson system. SIAM J. Math. Anal. 2019, 51, 3533-3568. [CrossRef]

24. Laskin, N. Fractional quantum mechanics and Lévy path integrals. Phys. Rev. A 2000, 268, 56-108. [CrossRef]

25. Bucur, C.; Valdinoci, E. Nonlocal Diffusion and Applications; Springer: Cham, Switzerland, 2016; Volume 20.

26. Di Nezza, E.; Palatucci, G.; Valdinoci, E. Hitchhiker's guide to the fractional Sobolev spaces. Bull. Sci. Math. 2012, 136, 521-573. [CrossRef]

27. Fröhlich, J.; Jonsson, B.L.G.; Lenzmann, E. Boson stars as solitary waves. Comm. Math. Phys. 2007, 274, 1-30. [CrossRef]

28. Kirkpatrick, K.; Lenzmann, E.; Staffilani, G. On the continuum limit for discrete NLS with long-range lattice interactions. Comm. Math. Phys. 2013, 317, 563-591. [CrossRef]

29. Klein, C.; Sparber, C.; Markowich, P. Numerical study of fractional nonlinear Schrödinger equations. Proc. R. Soc. A 2014, 470, 20140364. [CrossRef] [PubMed]

30. Longhi, S. Fractional Schrödinger equation in optics. Optics Lett. 2015, 40, 1117-1120. [CrossRef]

31. Metzler, R.; Klafter, J. The random walks guide to anomalous diffusion: A fractional dynamics approach. Phys. Rep. 2000, 339, 1-77. [CrossRef]

32. Vázquez, J.L. The mathematical theories of diffusion: Nonlinear and fractional diffusion. In Nonlocal and Nonlinear Diffusions and Interactions: New Methods and Directions; Springer: Cham, Switzerland; 2017; pp. 205-278.

33. Baleanu, D.; Etemad, S.; Rezapour, S. A hybrid Caputo fractional modeling for thermostat with hybrid boundary value conditions. Bound. Value Probl. 2020, 2020, 64. [CrossRef]

34. Baleanu, D.; Jajarmi, A.; Mohammadi, H.; Rezapour, S. A new study on the mathematical modelling of human liver with Caputo-Fabrizio fractional derivative. Chaos Solitons Fractals 2020, 134, 109705. [CrossRef]

35. Cabré, X.; Sire, Y. Nonlinear equations for fractional Laplacians, I: Regularity, maximum principles, and Hamiltonian estimates. Ann. Inst. H. Poincaré Anal. Non Linéaire 2014, 31, 23-53. [CrossRef]

36. Caffarelli, L.; Silvestre, L. An extension problem related to the fractional Laplacian. Comm. Partial Differ. Equ. 2007, 32, 1245-1260. [CrossRef]

37. Frank, R.L.; Lenzmann, E.; Silvestre, L. Uniqueness of radial solutions for the fractional Laplacian. Comm. Pure Appl. Math. 2016, 69, 1671-1726. [CrossRef]

38. Byeon, J.; Kwon, O.; Seok, J. Nonlinear scalar field equations involving the fractional Laplacian. Nonlinearity 2017, 30, 1659-1681. [CrossRef]

39. Chang, X.; Wang, Z.-Q. Ground state of scalar field equations involving a fractional Laplacian with general nonlinearities. Nonlinearity 2013, 26, 479-494. [CrossRef]

40. Felmer, P.; Quaas, A.; Tan, J. Positive solutions of the nonlinear Schrödinger equations with the fractional Laplacian. Proc. R. Soc. Edinb. Sect. A 2012, 142, 1237-1262. [CrossRef]

41. Ikoma, N. Existence of solutions of scalar field equations with fractional operator. J. Fixed Point Theory Appl. 2017, 19, 649-690. Erratum: J. Fixed Point Theory Appl. 2017, 19, 1649-1652. [CrossRef] 
42. Cingolani, S.; Gallo, M.; Tanaka, K. Normalized solutions for fractional nonlinear scalar field equation via Lagrangian formulation. Nonlinearity 2021, 34, 4017. [CrossRef]

43. Luo, H.; Zhang, Z. Normalized solutions to the fractional Schrödinger equations with combined nonlinearities. Calc. Var. Partial Differ. Equ. 2020, 59, 143. [CrossRef]

44. Lieb, E.H.; Yau, H.-T. The Chandrasekhar theory of stellar collapse as the limit of quantum mechanics. Comm. Math. Phys. 1987, 1121, 147-174. [CrossRef]

45. Argaez, C.; Melgaard, M. Solutions to quasi-relativistic multi-configurative Hartree-Fock equations in quantum chemistry. Nonlinear Anal. 2012, 75, 384-404. [CrossRef]

46. Dall'Acqua, A.; Østergaard Sørensen, T.; Stockmeyer, E. Hartree-Fock Theory for Pseudorelativistic Atoms. Ann. Henri Poincaré 2008, 9, 711-742. [CrossRef]

47. Cho, Y.; Fall, M.M.; Hajaiej, H.; Markowich, P.A.; Trabelsi, S. Orbital stability of standing waves of a class of fractional Schrödinger equations with Hartree-type nonlinearity. Anal. Appl. 2017, 15, 699-729. [CrossRef]

48. Lu, J.; Moroz, V.; Muratov, C.B. Orbital-free density functional theory of out-of-plane charge screening in graphene. J. Nonlinear Sci. 2015, 25, 1391-1430. [CrossRef]

49. D'Avenia, P.; Siciliano, G.; Squassina, M. On the fractional Choquard equations. Math. Models Methods Appl. Sci. 2015, 25, 1447-1476. [CrossRef]

50. Bhattara, S. On fractional Schrödinger systems of Choquard type. J. Diff. Equ. 2017, 263, 3197-3229. [CrossRef]

51. Gao, Z.; Tang, X.; Chen, S. Ground state solutions of fractional Choquard equations with general potentials and nonlinearities. Rev. R. Acad. Cienc. Exactas Fís. Nat. Ser. A Mat. RACSAM 2019, 113, 2037-2057. [CrossRef]

52. Luo, H. Ground state solutions of Pohozaev type for fractional Choquard equations with general nonlinearities. Comput. Math. Appl. 2019, 77, 877-887. [CrossRef]

53. Shen, Z.; Gao, F.; Yin, M. Ground state for nonlinear fractional Choquard equations with general nonlinearities. Math. Methods Appl. Sci. 2016, 39, 4082-4098. [CrossRef]

54. Berestycki, H.; Lions, P.-L. Nonlinear scalar field equations. I. Existence of a ground state. Arch. Ration. Mech. Anal. 1983, 82, 313-345. [CrossRef]

55. Hirata, J.; Tanaka, K. Nonlinear scalar field equations with $L^{2}$ constraint: Mountain pass and symmetric mountain pass approaches. Adv. Nonlinear Stud. 2019, 19, 263-290. [CrossRef]

56. Ikoma, N.; Tanaka, K. A note on deformation argument for $L^{2}$ constraint problems. Adv. Differ. Equ. 2019, 24, 609-646.

57. Jeanjean, L.; Tanaka, K. A remark on least energy solutions in $\mathbb{R}^{N}$. Proc. Am. Math. Soc. 2003, 131, 2399-2408. [CrossRef]

58. Feng, B.; Zhang, H. Stability of standing waves for the fractional Schrödinger-Hartree equation. J. Math. Anal. Appl. 2018, 460, 352-364. [CrossRef]

59. Lieb, E.H.; Seiringer, R.; Solovej, J.P.; Yngvason, J. The mathematics of the Bose gas and its condensation. In Oberwolfach Seminars; Birkäuser: Basel, Switzerland, 2005; Volume 34.

60. Pitaevskii, L.; Stringari, S. Bose-Einstein Condensation; Oxford University Press: Cambridge, MA, USA, 2003.

61. Lieb, E.H. Sharp constants in the Hardy-Littlewood-Sobolev and related inequalities. Ann. Math. 1983, 118, 349-374. [CrossRef]

62. Lions, P.-L. Symétrie et compacité dans les espaces de Sobolev. J. Funct. Anal. 1982, 49, 315-334. [CrossRef]

63. Cho, Y.; Ozawa, T. Sobolev inequalities with symmetry. Commun. Contemp. Math. 2009, 11, 355-365. [CrossRef]

64. Park, Y.J. Fractional Gagliardo-Nirenberg inequality. J. Chungcheong Math. Soc. 2011, 24, 583-586.

65. Palais, R.S. The principle of symmetric criticality. Comm. Math. Phys. 1979, 69, 19-30. [CrossRef]

66. Willem, M. Minimax theorems. In Progress in Nonlinear Differential Equations and Their Applications; Birkäuser: Boston, MA, USA, 1996; Volume 24.

67. Hirata, J.; Ikoma, N.; Tanaka, K. Nonlinear scalar field equations in $\mathbb{R}^{N}$ : Mountain pass and symmetric mountain pass approaches. Topol. Methods Nonlinear Anal. 2010, 35, 253-276.

68. Jeanjean, L. Existence of solutions with prescribed norm for semilinear elliptic equations. Nonlinear Anal. 1997, 28, 1633-1659. [CrossRef]

69. Dong, L.; Liu, D.; Qi, W.; Wang, L.; Zhou, H.; Peng, P.; Huang, C. Necklace beams carrying fractional angular momentum in fractional systems with a saturable nonlinearity. Commun. Nonlinear Sci. Numer. Simul. 2021, 99, 105840. [CrossRef]

70. Frank, R.L.; Lenzmann, E. On ground states for the $L^{2}$-critical boson star equation. arXiv 2010, arXiv:0910.2721.

71. Herr, S.; Lenzmann, E. The Boson star equation with initial data of low regularity. Nonlinear Anal. 2014, 97, 125-137. [CrossRef]

72. Lenzmann, E.; Lewin, M. On singularity formation for the $L^{2}$-critical Boson star equation. Nonlinearity 2011, 24, 3515-3540. [CrossRef] 